\title{
Introduction to the Notes
}

As explained in the introduction to the book, these notes are not fully worked 'model answers', which would be inappropriate in a collection of materials designed to be used flexibly in a variety of ways and at different levels to suit the needs of different courses and groups of students. The aim of the notes is to outline the main legal points which seem to emerge from the case studies, in order to provide a general framework within which the studies can be used and discussed.

In these notes, unless otherwise stated, references to section numbers are to the Companies Act 1985, and references to articles are to Table A in the Companies (Tables A to F) Regulations 1985. 